\title{
Analysis of a Mixed Finite Element Method for Elasto-Plastic Plates*
}

\author{
By F. Brezzi, C. Johnson and B. Mercier
}

\begin{abstract}
We consider a mixed finite element method for finding approximations of the displacement and moments in a thin elastic-perfectly plastic plate. Under some weak assumptions concerning the regularity of the exact solution, we prove an erior estimate for the moments.
\end{abstract}

1. Introduction. The purpose of this note is to prove an error estimate for a finite element method for thin elastic-perfectly plastic plates in the case of Hencky's law of plasticity. We shall consider a mixed finite element method, introduced by Herrmann [5] and Hellan [4] , based on a piecewise constant approximation of the moments and piecewise linear approximation of the displacements. An analysis of this method, in the case of elastic plates, can be found in Brezzi and Raviart [1] and Johnson [6]. The results of this note can be extended to the case of quasi-static evolution following the argument in Johnson [8]. For numerical results and practical experience of this method, we refer to Bäcklund [2].

We shall use the following notation: let $\Omega$ be a bounded Lipschitz region in $\mathbf{R}^{\mathbf{2}}$. For $k$ a natural number and $1 \leqslant q \leqslant \infty$, let $W^{k, q}$ be the Sobolev space with norm

$$
\|v\|_{k, q}=\left(\sum_{|\alpha| \leqslant k} \int_{\Omega}\left|D^{\alpha} v\right|^{q} d x\right)^{1 / q},
$$

with the usual modification if $q=\infty$. For $k=0$, we omit the subscript 0 and write $\|\cdot\|_{q}$ instead of $\|\cdot\|_{0, q}$. Let $H_{0}^{k}$ be the closure of $C_{0}^{\infty}(\Omega)$ with respect to the norm $\|\cdot\|_{k, 2}$. Let $H^{-1}=\left(H_{0}^{1}\right)^{\prime}$ be the dual of $H_{0}^{1}$ with duality pairing $\langle\cdot, \cdot\rangle$. Denote by $C(\bar{\Omega})$ the set of continuous functions on $\bar{\Omega}$ and $C_{0}(\bar{\Omega})=\{v \in C(\bar{\Omega}): v=0$ on $\partial \Omega\}$, and let $M(\Omega)=\left(C_{0}(\bar{\Omega})\right)^{\prime}=$ bounded measures on $\bar{\Omega}$ \} be the dual of $C_{0}(\bar{\Omega})$ with duality pairing $[\cdot, \cdot]$.

Let $x=\left(x_{1}, x_{2}\right)$ denote the coordinates in $\mathbf{R}^{2}$ and set

$$
v_{, i}=\frac{\partial v}{\partial x_{i}}, \quad v_{, i j}=\frac{\partial^{2} v}{\partial x_{i} \partial x_{j}}, \quad i, j=1,2 .
$$

We shall use the convention that repeated indices indicate summation from 1 to 2 .

2. The Plasticity Problem. Consider a clamped isotropic elastic-perfectly plastic plate occupying the region $\Omega$ in $\mathbf{R}^{2}$, subject to a transversal load $f \in L^{2}(\Omega)$. Suppose that the set of plastically admissible moments $\tau=\left\{\tau_{i j}\right\}, i, j=1,2$, is given by $B=$ $\left\{\tau \in \mathbf{R}^{4}: F(\tau) \leqslant 1\right\}$, where $F: \mathbf{R}^{4} \rightarrow \mathbf{R}$ is continuous, convex and $F(0)=0$.

Received September 21, 1976.

AMS (MOS) subject classifications (1970). Primary 65N15.

*This work has been performed during the stay of F. Brezzi and C. Johnson in the Centre de Mathématiques Appliquées of Ecole Polytechnique (Palaiseau). 
Since the plate is isotropic, we may assume that $F$ is invariant under changes of coordinates, i.e., $F(\tau)$ depends only on the eigenvalues of the symmetric matrix $\left(\tau_{i j}\right)$. Define

$$
\begin{aligned}
H & =\left\{\tau=\left\{\tau_{i j}\right\}: \tau_{i j} \in L^{2}(\Omega), i, j=1,2, \tau_{12}=\tau_{21}\right\}, \\
(\sigma, \tau) & =\int_{\Omega} \sigma_{i j} \tau_{i j} d x, \quad\|\tau\|=(\tau, \tau)^{1 / 2}, \quad \sigma, \tau \in H, \\
(v, w) & =\int_{\Omega} v w d x, \quad\|v\|=(v, v)^{1 / 2}, \quad v, w \in L^{2}(\Omega), \\
P & =\{\tau \in H: \tau(x) \in B \text { a.e. in } \Omega\}, \\
D \tau & =\tau_{i j, i j}, \quad \widetilde{P}=\left\{\tau \in P: D \tau \in H^{-1}\right\}, \\
E(t) & =\{\tau \in H: D \tau=t f\}, \quad t \geqslant 0, \\
K(t) & =E(t) \cap P, \quad E=E(1), \quad K=K(1) .
\end{aligned}
$$

We note the following Green's formula: For $v \in H_{0}^{2}$, and $\tau \in H$ with $D \tau \in L^{2}(\Omega)$, one has

$$
(v, D \tau)=(\epsilon(v), \tau)
$$

where

$$
\epsilon(v)=\left\{v_{, i j}\right\}
$$

The problem of finding the moments $\sigma$ and displacements $u$ in the elasto-plastic plate can be formulated as follows:

Find $(\sigma, u) \in \widetilde{P} \times H_{0}^{1}$ such that

$$
\begin{aligned}
& (\sigma, \tau-\sigma)-\langle u, D \tau-D \sigma\rangle \geqslant 0, \quad \tau \in \widetilde{P}, \\
& \langle v, D \sigma\rangle=(v, f), \quad v \in H_{0}^{1} .
\end{aligned}
$$

Existence of a solution of this problem can be proved under the following hypothesis:

$$
\text { There exists } \chi \in E \text { and } \delta>0 \text { such that }(1+\delta) \chi \in P \text {. }
$$

Remark. Note that $(\mathrm{H}-1) \Longleftrightarrow K(1+\delta) \neq \varnothing$. On the other hand, $K(1+\delta) \neq \varnothing$ for some $\delta>0$ means that $f$ is not a limit load (see [10]). Thus (H-1) is a "safe load hypothesis".

TheOREM 1. If $(\mathrm{H}-1)$ holds, then there exists $(\sigma, u) \in \widetilde{P} \times H_{0}^{1}$ satisfying (2.2). Moreover, $\sigma$ is uniquely determined.

Remark 2. One can also show that $u \in L^{\infty}(\Omega)$.

Proof of Theorem 1. The uniqueness of $\sigma$ follows easily. To prove the existence, we shall apply the technique used in [7]. For $\nu, \mu>0$, we consider the following problem obtained by penalizing the constraint $\sigma \in P$ and regularizing with respect to $u$ : Find $\left(\sigma_{\mu \nu}, u_{\mu \nu}\right) \in H \times H_{0}^{2}$ such that

$$
\left(\sigma_{\mu \nu}, \tau\right)+\left(J_{\mu}^{\prime}\left(\sigma_{\mu \nu}\right), \tau\right)-\left(\epsilon\left(u_{\mu \nu}\right), \tau\right)=0, \quad \tau \in H
$$




$$
\nu\left(\epsilon(v), \epsilon\left(u_{\mu \nu}\right)\right)+\left(\epsilon(v), \sigma_{\mu \nu}\right)=(v, f), \quad v \in H_{0}^{2},
$$

where

$$
J_{\mu}^{\prime}(\tau)=\frac{1}{\mu}(\tau-\pi \tau)
$$

is the Gateaux derivative of

$$
J_{\mu}(\tau)=\frac{1}{2 \mu}\|\tau-\pi \tau\|^{2}, \quad \tau \in H,
$$

and $\pi: \mathbf{R}^{4} \rightarrow \mathbf{R}^{4}$ is the orthogonal projection onto $B$.

Using a result $[3$, p. 161] on the existence of saddle points, we see that this problem has a solution. Taking $\tau=\sigma_{\mu \nu}-\chi$ in (2.3a) and $v=u_{\mu \nu}$ in (2.3b), and adding, we find easily that

$$
1 / 2\left\|\sigma_{\mu \nu}\right\|^{2}+\nu\left\|\epsilon\left(u_{\mu \nu}\right)\right\|^{2}+\left(J_{\mu}^{\prime}\left(\sigma_{\mu \nu}\right), \sigma_{\mu \nu}-\chi\right) \leqslant 1 / 2\|\chi\|^{2},
$$

using the fact that $\chi \in E$. Since $J_{\mu}(\chi)=0$ and by the convexity of $J_{\mu}$,

$$
J_{\mu}\left(\sigma_{\mu \nu}\right)-J_{\mu}(\chi) \leqslant\left(J_{\mu}^{\prime}\left(\sigma_{\mu \nu}\right), \sigma_{\mu \nu}-\chi\right),
$$

we thus obtain the a priori estimates

$$
\begin{gathered}
\left\|\sigma_{\mu \nu}\right\|^{2} \leqslant C, \\
\sqrt{\nu}\left\|\epsilon\left(u_{\mu \nu}\right)\right\| \leqslant C, \\
J_{\mu}\left(\sigma_{\mu \nu}\right) \leqslant\left(J_{\mu}^{\prime}\left(\sigma_{\mu \nu}\right), \sigma_{\mu \nu}-\chi\right) \leqslant C,
\end{gathered}
$$

where $C$ does not depend on $\mu$ and $\nu$.

Next, using (H-1) as in [7], we see that there exists a positive constant $\beta$ depending only on $\delta$ and $B$ such that

$$
\beta\left\|J_{\mu}^{\prime}\left(\sigma_{\mu \nu}\right)\right\|_{1} \leqslant\left(J_{\mu}^{\prime}\left(\sigma_{\mu \nu}\right), \sigma_{\mu \nu}-\chi\right) \leqslant C .
$$

Thus, by (2.3a) and (2.5), we have $\left\|\epsilon\left(u_{\mu \nu}\right)\right\|_{1} \leqslant C$, so that by Sobolev's embedding theorem,

$$
\left\|u_{\mu \nu}\right\|_{1,2} \leqslant C \text {. }
$$

Letting first $\nu$ and then $\mu$ tend to zero, using (2.5)-(2.8) and (2.1), we finally obtain $(\sigma, u) \in \widetilde{P} \times H_{0}^{1}$ satisfying (2.2). This completes the proof of the theorem.

The above existence result is slightly too weak to permit a discretization using the mixed finite element of Herrmann. To this end, we shall need the following hypothesis concerning the function $u \in H_{0}^{1} \cap L^{\infty}(\Omega)$ given by Theorem 1:

$$
u \in C_{0}(\bar{\Omega}) \text {. }
$$

Remark. Note that it is not true in general that $u \in H_{0}^{2}$ since $u_{, i}$ may be discontinuous. To indicate what can happen, we give the following one dimensional example:

$$
\Omega=]-1,1\left[, \quad \epsilon \equiv \frac{d^{2}}{d x^{2}}, \quad B=\{\sigma \in \mathbf{R}|| \sigma \mid \leqslant 1\}, \quad f=\right.\text { constant. }
$$

We check that, for $0 \leqslant f \leqslant 3$, the solution is elastic 
and that, for $3<f \leqslant 4$, the solution is elastic-plastic

$$
u=\frac{f}{24}(1-x)^{2}(1+x)^{2}, \quad \sigma=\frac{f}{2}\left(x^{2}-\frac{1}{3}\right)
$$

$$
u=\frac{f}{24}\left(1-x^{2}\right)\left(c-x^{2}\right) \notin H_{0}^{2}(\Omega), \quad \sigma=\frac{f}{2}\left(x^{2}-b\right),
$$

where $c=5-12 / f$ and $b=1-2 / f$. Note that at points $x$ (unknown beforehand) where $|\sigma(x)|=1$, the control of $d u / d x$ is lost; in the example, the boundary condition $d u( \pm 1) / d x=0$ is not satisfied for $3<f \leqslant 4$. More generally, $d u / d x$ may be discontinuous at points where the plasticity condition $|\sigma(x)|=1$ is active.

3. Approximation. For the finite element approximation we shall use two finite dimensional spaces $V_{h}$ and $P_{h}$ depending on the positive parameter $h$, such that $V_{h} \subset$ $C_{0}(\bar{\Omega})$ and $P_{h} \subset P$. For $\tau \in P_{h}, D \tau$ will be a finite sum of Dirac measures, so that $D \tau \in M(\Omega)$ but $D \tau \notin H^{-1}$. Thus, in the discrete problem, we shall choose to work with the duality $[\cdot, \cdot]$ between $C_{0}(\bar{\Omega})$ and $M(\Omega)$ instead of the duality $\langle\cdot, \cdot\rangle$ between $H_{0}^{1}$ and $H^{-1}$ used in the continuous problem. This will make sense since $V_{h} \subset C_{0}(\bar{\Omega})$ but will eventually call for assumption $(\mathrm{H}-2)$.

Let us now define the spaces $V_{h}$ and $P_{h}$. For simplicity, we shall assume that $\Omega$ is a convex polygonal domain. For $0<h<1$, let $T_{h}=\{T\}$ be a triangulation of $\Omega$ with $h=\max \left\{\operatorname{diam} T: T \in T_{h}\right\}$. We shall assume as usual that $T_{h}$ is regular, i.e., there exists a constant $\theta>0$, independent of $h$, such that for $T \in T_{h}$, the minimum angle of $T$ is greater than $\theta$. Define

$$
\begin{aligned}
& V_{h}=\left\{v \in C_{0}(\bar{\Omega}):\left.v\right|_{T} \text { is linear in } T, T \in T_{h}\right\}, \\
& H_{h}=\left\{\tau \in H:\left.\tau\right|_{T} \text { is constant in } T, T \in T_{h}\right\} .
\end{aligned}
$$

In order to define $P_{h}$, we note the following Green's formula: if $\left.v\right|_{T} \in C^{\infty}(T)$, $T \in T_{h}$, and $\tau \in H_{h}$, then

$$
\sum_{T} \int_{T} v_{, i j} \tau_{i j} d x=\sum_{T}\left\{-\int_{T} v_{, i} \tau_{i j, j} d x+\int_{\partial T} M_{n}(\tau) \frac{\partial v}{\partial n} d s+\int_{\partial T} M_{n t}(\tau) \frac{\partial v}{\partial t} d s\right\}
$$

where for $T \in T_{h}$,

$$
M_{n}(\tau)=\tau_{i j} n_{i} n_{j}, \quad M_{n t}(\tau)=\tau_{i j} n_{i} t_{j},
$$

where $n=\left(n_{1}, n_{2}\right)$ is the unit outward normal to $\partial T$ and $t=\left(n_{2},-n_{1}\right)$, is the unit tangent along $\partial T$. For $\tau \in H_{h}$, we shall say that $M_{n}(\tau)$ is continuous at the interelement boundaries if for $T^{\prime}, T^{\prime \prime} \in T_{h}$ with $T^{\prime} \cap T^{\prime \prime} \neq \varnothing$, one has

$$
M_{n^{\prime}}\left(\left.\tau\right|_{T^{\prime}}\right)=M_{n^{\prime \prime}}\left(\left.\tau\right|_{T^{\prime \prime}}\right) \quad \text { on } T^{\prime} \cap T^{\prime \prime}
$$

where $n^{\prime}$ and $n^{\prime \prime}$ are the unit outward normals to $T^{\prime}$ and $T^{\prime \prime}$, respectively. We now define $P_{h}$ as

$$
P_{h}=M_{h} \cap P
$$

where

$$
M_{h}=\left\{\tau \in H_{h}: M_{n}(\tau) \text { is continuous at interelement boundaries }\right\}
$$


As the degrees of freedom for $M_{h}$ one can take the values of $M_{n}(\tau)$ at the midpoint of each side of $T_{h}$. Let us note that for $v \in C_{0}^{\infty}(\Omega)$ and $\tau \in M_{h}$, the Green's formula (3.1) reduces to

$$
(\epsilon(v), \tau)=\sum_{T} \int_{\partial T} M_{n t}(\tau) \frac{\partial v}{\partial t} d s,
$$

since $\tau_{i j, j}=0$ in each $T$ and $M_{n}(\tau)$ is continuous at interelement boundaries. Further, integrating by parts along each side of each $T \in T_{h}$, we find that (3.2) can be written

$$
(\epsilon(v), \tau)=\sum_{N \in N_{h}} R(\tau ; N) v(N), \quad v \in C_{0}^{\infty}(\Omega), \tau \in M_{h},
$$

where $N_{h}=\{N\}$ is the set of nodes of $T_{h}$ and for $N \in N_{h}, R(\tau ; N)$ is a certain linear combination of the values of $\tau$ for the triangles meeting at $N$. Since $C_{0}^{\infty}(\Omega)$ is dense in $C_{0}(\bar{\Omega}),(3.3)$ implies:

Lemma 1. If $\tau \in M_{h}$, then $D \tau \in M(\Omega)$ and

$$
[v, D \tau]=\sum_{N} R(\tau ; N) v(N), \quad v \in C_{0}(\bar{\Omega}) .
$$

Let us now formulate the discrete problem:

Find $\left(\sigma_{h}, u_{h}\right) \in P_{h} \times V_{h}$ such that

$$
\begin{gathered}
\left(\sigma_{h}, \tau-\sigma_{h}\right)-\left[u_{h}, D \tau-D \sigma_{h}\right] \geqslant 0, \quad \tau \in P_{h}, \\
{\left[v, D \sigma_{h}\right]=(v, f), \quad v \in V_{h} .}
\end{gathered}
$$

Note that $\left(\sigma_{h}, u_{h}\right)$ can be equivalently characterized as a saddle point of the functional $L: P_{h} \times V_{h} \rightarrow \mathrm{R}$ defined by

$$
L(\tau, v)=1 / 2\|\tau\|^{2}-[v, D \tau]+(v, f) .
$$

Existence of a solution of (3.4) then follows (see, e.g. [12, Section 28]) from the following qualification hypothesis corresponding to $(\mathrm{H}-1)$ :

$$
\text { There exists } \delta>0 \text { and } \chi_{h} \in E_{h} \text { such that }(1+\delta) \chi_{h} \in P \text {, and }\left\|\chi_{h}\right\| \leqslant C,
$$

where

$$
E_{h}=\left\{\tau \in M_{h}:[v, D \tau]=(v, f), v \in V_{h}\right\},
$$

and $C$ and $\delta$ do not depend on $h$.

If $\chi$ is sufficiently regular (e.g. $\chi \in\left[W^{1,1} \cap C(\bar{\Omega})\right]^{4}$ ), then (H-1) will imply (H-3). To see this, we introduce the interpolation operator $\pi_{h}:\left[W^{1,1} \cup C(\bar{\Omega})\right]^{4} \rightarrow M_{h}$, defined by

$$
\left.M_{n}\left(\pi_{h} \tau\right)\right|_{S}=\frac{1}{|S|} \int_{S} M_{n}(\tau) d s, \quad S \in S_{h}
$$

where $S_{h}=\{S\}$ denotes the sides of the triangles $T \in T_{h}$ and $|S|=$ length of $S$. We note the following important property of $\pi_{h}$ :

Lemma 2. If $\tau \in\left[W^{1,1}\right]^{4}$ and $D \tau \in L^{2}(\Omega)$, then

$$
(v, D \tau)=\left[v, D \pi_{h} \tau\right], \quad v \in V_{h} .
$$


Proof. Integrating by parts over $\Omega$, we have, if $v \in V_{h}$,

$$
(v, D \tau)=-\int_{\Omega} v_{, i} \tau_{i j, j} d x
$$

By (3.1) we also have

$$
\begin{aligned}
-\int_{\Omega} v_{, i} \tau_{i j, j} d x & =\sum_{T}\left\{\int_{T} v_{, i j} \tau_{i j} d x-\int_{\partial T} M_{n}(\tau) \frac{\partial v}{\partial n} d s-\int_{\partial T} M_{n t}(\tau) \frac{\partial v}{\partial t} d s\right\} \\
& =-\sum_{T} \int_{\partial T} M_{n}(\tau) \frac{\partial v}{\partial n} d s,
\end{aligned}
$$

since $v_{, i j}=0$ in each $T \in T_{h}$ and $M_{n t}(\tau)$ takes the same values on adjacent sides $S$. By the definition of $\pi_{h} \tau$ and since $\partial v / \partial n$ is constant on each side $S$, we thus have

$$
(v, D \tau)=-\sum_{T} \int_{\partial T} M_{n}\left(\pi_{h} \tau\right) \frac{\partial v}{\partial n} d s, \quad v \in V_{h} .
$$

Finally, using (3.1), as we know for all $\widetilde{\tau} \in M_{h}, v \in V_{h}$,

$$
-\sum_{T} \int_{\partial T} M_{n}(\widetilde{\tau}) \frac{\partial v}{\partial n} d s=\sum_{T} \int_{\partial T} M_{n t}(\tilde{\tau}) \frac{\partial v}{\partial t} d s
$$

so that

$$
(v, D \tau)=\sum_{T} \int_{\partial T} M_{n t}\left(\pi_{h} \tau\right) \frac{\partial v}{\partial t} d s=\sum_{N} R\left(\pi_{h} \tau ; N\right) v(N)=\left[v, D \pi_{h} \tau\right], \quad v \in V_{h},
$$

which completes the proof of the lemma.

Corollary 1. If $\tau \in\left[W^{1,1}\right]^{4} \cap E$, then $\pi_{h} \tau \in E_{h}$.

Corollary 2. If $\chi \in\left[W^{1,1} \cap C(\bar{\Omega})\right]^{4}$, then $(\mathrm{H}-1)$ implies $(\mathrm{H}-3)$.

Proof. Choosing $\chi_{h}=\pi_{h} \chi$, we have $\chi_{h} \in E_{h}$. Further, by standard interpolation theory (cf. [1] , [6] ), $\left\|\chi-\chi_{h}\right\|_{\infty} \rightarrow 0$ as $h \rightarrow 0$, which proves the desired result.

The error estimate will be expressed in terms of the quantity

$$
\alpha(h)=\inf \left\{\alpha>0: \exists \tau \in E_{h} \text { with }\|\sigma-\tau\| \leqslant \alpha \text { and }(1-\alpha) \tau \in P\right\},
$$

where we assume, to make what follows of any interest, that, for some constant $C$, $\alpha(h) \leqslant C$. The quantity $\alpha(h)$ is a measure of how well the exact solution $\sigma \in E \cap P$ can be approximated by functions $\tau \in E_{h}$ "almost" satisfying $\tau \in P$. As above, for $\sigma$ sufficiently regular (e.g. $\sigma \in\left[W^{1,1} \cap C(\bar{\Omega})\right]^{4}$ ), choosing $\tau=\pi_{h} \sigma$ in (3.5), we see that $\alpha(h) \rightarrow 0$ as $h \rightarrow 0$. Let us now state the main result of this note.

THEOREM 2. If hypotheses (H-1)-(H-4) hold (for (H-4), see Lemma 4 below), then there exists a constant $C$ independent of $h$, such that for $h$ sufficiently small,

$$
\left\|\sigma-\sigma_{h}\right\| \leqslant C(\alpha(h)+h)^{1 / 2} .
$$

To prove this estimate, we shall need the following two lemmas. By $C$ we will denote a positive constant, independent of $h$, not necessarily the same at each occurrence.

LEMм A 3. If (H-3) holds, then

(i) $\left\|\sigma_{h}\right\| \leqslant C$,

(ii) $\left\|u_{h}\right\| \leqslant C$. 
Proof. Taking $\tau=\chi_{h}$ in (3.4a), we obtain

$$
\left\|\sigma_{h}\right\|^{2} \leqslant\left(\sigma_{h}, \chi_{h}\right) \leqslant\left\|\sigma_{h}\right\|\left\|\chi_{h}\right\| \leqslant C\left\|\sigma_{h}\right\|
$$

which proves (i). To prove (ii), we shall first establish the following stability result: there exists a constant $C$ such that

$$
\|v\| \leqslant C \sup _{\tau \in M_{h}} \frac{[v, D \tau]}{\|\tau\|_{\infty}}, \quad v \in V_{h} .
$$

To prove (3.6), let $v \in V_{h}$ and let $\psi \in H_{0}^{2}$ satisfy $\Delta^{2} \psi=v$ in $\Omega$. Since $\Omega$ is a convex polygon, we then have $\|\psi\|_{3+\epsilon, 2} \leqslant C\|v\|$ for some $\epsilon>0$ (see [9]), so that by Sobolev's embedding theorem with $\tau=\left\{\psi_{, i j}\right\}$,

$$
\|\tau\|_{\infty} \leqslant C\|v\|,
$$

with $C$ independent of $v$. We have, by Lemma 2,

$$
\|v\|^{2}=(v, D \tau)=\left[v, D \pi_{h} \tau\right]
$$

so that, by (3.7) and the easily established fact that $\left\|\pi_{h} \tau\right\|_{\infty} \leqslant C\|\tau\|_{\infty}$ (note that $\tau$ is continuous), one has

$$
\|v\| \leqslant C \frac{\left[v, D \pi_{h} \tau\right]}{\|\tau\|_{\infty}} \leqslant C \frac{\left[v, D \pi_{h} \tau\right]}{\left\|\pi_{h} \tau\right\|_{\infty}}
$$

which proves (3.6). Next, we note that by (H-3), there exists a constant $\widetilde{\delta}>0$ such that

$$
x_{h}+\tilde{\tau} \in P_{h} \quad \text { if }\|\tilde{\tau}\|_{\infty} \leqslant \tilde{\delta}, \tilde{\tau} \in M_{h}
$$

Taking $\tau=\chi_{h}+\tilde{\tau}$ in (3.4a), we see that since $\left[u_{h}, D \chi_{h}-D \sigma_{h}\right]=0$,

$$
\left[u_{h}, D \tilde{\tau}\right] \leqslant\left(\sigma_{h}, \chi_{h}+\tilde{\tau}-\sigma_{h}\right) .
$$

By (i), we thus have

$$
\left[u_{h}, D \tilde{\tau}\right] \leqslant C \text { if } \tilde{\tau} \in M_{h},\|\tilde{\tau}\|_{\infty} \leqslant \widetilde{\delta},
$$

which together with (3.6) proves (ii).

In the proof of Theorem 2, we shall need to take $\tau=\sigma_{h}$ in (2.2a). Since $P_{h} \not \subset$ $\widetilde{P}$, this has to be justified. We have

LEMMA 4. If (H-2) holds and

$$
\tau \in B \Rightarrow\left(\tau_{11}, 0,0\right) \in B
$$

then

$$
(\sigma, \tau-\sigma)-[u, D \tau-D \sigma] \geqslant 0, \quad \tau \in P_{h} .
$$

Remark. (H-4) is needed only in order to construct a suitable extension of $\sigma_{h}$ outside $\Omega$. It is likely that this extension can be made even without assuming ( $\mathrm{H}-4)$, but we do not know how to make the construction in this case. Let us note that (H-4) is satisfied in particular in the case of Tresca's yield criterion for metal plates, i.e., in 
the case when

$$
\tau \in B \Longleftrightarrow \max \left(\left|\lambda_{1}\right|,\left|\lambda_{2}\right|,\left|\lambda_{1}-\lambda_{2}\right|\right) \leqslant 1,
$$

or in the case of Johansson's yield criterion for reinforced concrete plates, i.e.,

$$
\tau \in B \Longleftrightarrow \max \left(\left|\lambda_{1}\right|,\left|\lambda_{2}\right|\right) \leqslant 1,
$$

where $\lambda_{1}$ and $\lambda_{2}$ are the eigenvalues of the symmetrix matrix $\left(\tau_{i j}\right)$ (see [10]).

Proof of Lemma 4. Let $\tau \in P_{h}$ be given. Using (H-4), we first construct an extension $\tilde{\tau}$ of $\tau$ to a polygonal domain $\widetilde{\Omega} \supset \bar{\Omega}$ in such a way that $\tilde{\tau}(x) \in B, x \in \widetilde{\Omega}$, and $D \tilde{\tau} \in M(\widetilde{\Omega})$ (for details, see [11]). Then, we regularize $\tilde{\tau}$ by convolving with a smooth function $\varphi_{r}$ defined by $\varphi_{r}(x)=r^{-2} \varphi(x / r)$, where $\varphi \in C_{0}^{\infty}\left(R^{2}\right), \varphi$ is positive and the mean value of $\varphi$ is equal to one. For the regularized function, we can then use (2.2a); and passing to the limit as $r \rightarrow 0$, we obtain (3.8). We leave the details to the interested reader.

Proof of Theorem 2. For $\alpha>\alpha(h)$, there exists $\tau_{h} \in E_{h}$ such that $\left\|\sigma-\tau_{h}\right\| \leqslant \alpha$ and $(1-\alpha) \tau_{h} \in P_{h}$. Taking $\tau=(1-\alpha) \tau_{h}$ in (3.4a), $\tau=\sigma_{h}$ in (3.8), and adding, we get

$$
\begin{aligned}
\left\|\sigma-\sigma_{h}\right\|^{2} & \leqslant\left(\sigma_{h},(1-\alpha) \tau_{h}-\sigma\right)+\left[u_{h}, D \sigma_{h}-(1-\alpha) D \tau_{h}\right]+\left[u, D \sigma-D \sigma_{h}\right] \\
& \equiv A_{1}+A_{2}+A_{3}
\end{aligned}
$$

with obvious notation. We have by Lemma 3 ,

$$
A_{1} \leqslant\left\|\sigma_{h}\right\|\left\|\sigma-\tau_{h}\right\|+\alpha\left\|\sigma_{h}\right\|\left\|\tau_{h}\right\| \leqslant C \alpha .
$$

Further, since $\sigma_{h}$ and $\tau_{h} \in E_{h}$, we have by Lemma 3,

$$
A_{2}=\alpha\left(u_{h}, f\right) \leqslant C \alpha .
$$

Finally, to estimate $A_{3}$, we note that by (3.4b),

$$
R\left(\sigma_{h} ; N\right)=\left(\varphi_{N}, f\right), \quad N \in N_{h},
$$

where $\varphi_{N} \in V_{h}$ is the basis function which takes the value 1 at node $N$ and vanishes at the other nodes. Thus, by Lemma 1 ,

$$
\left[u, D \sigma_{h}\right]=\sum_{N} R\left(\sigma_{h} ; N\right) u(N)=\sum_{N} u(N)\left(\varphi_{N}, f\right)=\left(\tilde{u}_{h}, f\right),
$$

where $\tilde{u}_{h} \in V_{h}$ interpolates $u$ at the nodes $N_{h}$. Therefore, by well-known interpolation theory,

$$
A_{3}=\left(\tilde{u}_{h}-u, f\right) \leqslant\|f\|\left\|u-\tilde{u}_{h}\right\| \leqslant C h\|f\|\|u\|_{1,2} \leqslant C h .
$$

Combining (3.9)-(3.12), we then obtain the statement of the theorem.

Corollary. If $(\mathrm{H}-1)-(\mathrm{H}-4)$ hold and $\sigma \in W^{1,1} \cap C(\bar{\Omega})$, then $\left\|\sigma-\sigma_{h}\right\| \rightarrow 0$ as $h \longrightarrow 0$. 
Institutionen för Informationsbehandling

Chalmers T. H.

Fack 40220 Göteborg, Sweden

École Poly technique

Centre de Mathématiques Appliquées

Route de Saclay

91120 Palaiseau, France

1. F. BREZZI \& P. A. RAVIART, "Mixed finite element methods for 4th order problems," Topics in Numerical Analysis. III, J. MILLER (Editor), Academic Press, New York, 1976.

2. J. BÄCKLUND, "Mixed finite element analysis of elasto-plastic plates in bending," Arch. Mech., v. 24, 1972, pp. 319-335.

3. I. EKELAND \& R. TEMAM, Analyse Convexe et Problèmes Variationnels, Dunod, Paris, 1973.

4. K. HELLAN, "An analysis of elastic plates in flexure by a simplified finite element method," Acta Polytech. Scand. Ci. Ser., v. 46, 1967.

5. L. R. HER RMANN, "Finite element bending analysis for plates," J. Engr. Mech. Div. $A S C E$, EM5, a3, 1967, pp. 49-83.

6. C. JOHNSON, "On the convergence of some mixed finite element methods for plate bending problems," Numer. Math., v. 21, 1973, pp. 43-62.

7. C. JOHNSON, "Existence theorems for plasticity problems," J. Math. Pures Appl., v. 55, 1976, pp. $431-444$.

8. C. JOHNSON, "A mixed finite element method for plasticity with hardening," SIAM J. Numer. Anal. (To appear.)

9. V. A. KONDRAT'EV, "Boundary value problems for elliptic equations in domains with conical or angular points," Trudy Moskov. Mat. Obక̌c., v. 16, 1967, pp. 209-292. (Russian) MR 37 \#1777.

10. C. E. MASSONNET \& M. A. SAVE, Plastic Analysis and Design of Plates, Shells and Disks, North-Holland, Amsterdam, 1972.

11. B. MERCIER, Sur la Théorie et l'Analyse Numérique de Problèmes de Plasticité, Thèse, Paris, 1977.

12. R. T. ROCKAfEllar, Convex Analysis, Princeton Univ. Press, Princeton, N. J., 1970. MR $43 \# 445$. 